Vol. 18,\#6, Junio 2010

http:// revista-redes.rediris.es

\title{
Réseaux relationnels et processus de soutien aux malades de la tuberculose au Cameroun
}

\author{
Yves Bertrand Djouda Feudjio, Sociologue - Université de Yaoundé I $^{1}$
}

\begin{abstract}
Resumé
Nombre de malades en Afrique ne jouissent pas d'une réelle autonomie dans le choix de leur trajectoire thérapeutique. La maladie d'un individu implique les principaux membres de son entourage socio-familial. Elle concerne aussi ses cousines, ses tantes, ses parents, ses amis et ses voisins. Ce " groupe organisateur profane de la thérapie » décide non seulement du moment où le malade doit visiter I'hôpital ou le guérisseur, mais, il joue un rôle crucial pour ce qui est de la détermination du diagnostic, de la trajectoire du malade et de la prise des décisions afférentes. La trajectoire thérapeutique suivie peut n'être pas celle voulue par le malade mais celle choisie par son réseau relationnel. Les choix thérapeutiques ne sont donc pas toujours individuels ou isolés, ils ont une histoire et sont déterminés par un « habitus » familial ou par les rapports de force socio-familiaux. Bien que la tuberculose (TB) soit restée une maladie stigmatisante, la prise en charge des malades tuberculeux au Cameroun reste influencée et déterminée par ses contraintes socio-familiales.
\end{abstract}

Mots clés: Réseaux familiaux, processus de soutien, malades tuberculeux, Cameroun.

\begin{abstract}
A number of patients in Africa do not enjoy real autonomy in choosing their treatment path. The disease involves an individual senior member of his entourage socio-familial. She also relates her cousins, aunts, parents, friends and neighbors. This "secular organization group therapy" decides not only when the patient must visit the hospital or healer, but it plays a crucial role with regard to determining the diagnosis, the trajectory of the patient and making decisions related. The trajectory followed by treatment that may not be desired by the patient but one chosen by the network of relationships. The therapeutic choices are not always individual or isolated; they have a history and are determined by habitus or family power relations. Although tuberculosis (TB) has remained a stigmatizing disease, the treatment of tuberculosis patients in Cameroon is influenced and determined by his social and family constraints.
\end{abstract}

Keywords: family networks, processes and support, TB patients, Cameroon.

\footnotetext{
${ }^{1}$ Enviar correspondencia a: [feudjioyb@yahoo.fr].
} 


\section{I ntroduction}

La tuberculose (TB) a refait surface et est devenue un énorme « fléau, un énorme « fardeau ${ }^{3}$ » et « une maladie bien vivante ${ }^{4}$ » dans toute la région africaine. Avec seulement $11 \%$ de la population mondiale, le continent Afrique porte plus d'un quart $(27 \%)$ du poids mondial de la TB et marque ainsi une réelle « urgence ${ }^{5}$ ». «Malgré des efforts louables de la part des pays et des partenaires dans la lutte contre la TB, il $n$ y a pas eu d'effets significatifs sur l'épidémie qui a pris une ampleur sans précédant [et nécessite] des mesures d'urgence extraordinaires. ${ }^{6}$ » Au Cameroun, la situation épidémiologique de l'endémie TB est préoccupante. Le pays reste une zone de forte endémie tuberculose ${ }^{7}$. Alors qu'en 1994 on pouvait compter seulement 18000 personnes souffrant de la TB à microspie positive, aujourd'hui, l'on enregistre un ratio d'au moins 300 cas pour 100000 habitants. Le taux de guérison qui avait atteint le pic de $73 \%$ en 2000 a rechuté et n'a cessé de décroître. En 2003, il était retombé à 63\%. Le taux des malades perdus de vue est préoccupant. II est passé de $11 \%$ en 1999 à près de $20 \%$ ces dernières années ${ }^{8}$. Les districts de santé des zones périphériques du pays présentent une situation épidémiologique plus préoccupante encore. Le $\mathrm{CDT}^{9}$ de I'hôpital de district de Foumban par exemple, qui recrutait dans les années 1997 et 1998 moins de 50 malades tuberculeux par an, cumule depuis l'année 2000, au moins 100 malades tuberculeux chaque année. Ce CDT a cumulé jusqu'ici au moins 70 cas de retraitement, ce qui signifie que la prise en charge thérapeutique de la pandémie pose des questionnements qui méritent d'être explorés. Les efforts institutionnels

\footnotetext{
2 Aubey, P., « La tuberculose à l'heure du Sida, actualités 2004 », in Médecine tropicale, disponible sur http://medecinetropicale.free.frl.

${ }^{3}$ Murray, C. J. L. et al., «La tuberculose dans les pays en développement: importance, stratégies de lutte et coût », in Bulletin de l'Union internationale contre la tuberculose et les maladies respiratoires, vol. 65, no 1, 1990.

${ }^{4}$ Brown, P., « Une maladie bien vivante », in Santé du monde, no 4, juillet-août, 1993, p.4-5.

${ }^{5}$ Consulter le site http://www.ulb.ac. be/esprit libre/ (esprit libre, juin 2003).

${ }^{6}$ Propos de Luis Gomes Sambo, tenus le 4 mai 2005 à Addis Abeba, au cours d'une session spéciale sur la lutte contre la TB dans la région africaine. II était alors directeur régional de I'OMS pour l'Afrique. Disponible sur http// : www. populationdata. net/sante/afrique-tuberculose-sept 05.

7 Berçon, R. et Kuaban, C., Résistances de mycrobactérium tuberculosis complex aux principaux antibacillaires à Yaoundé, 1988.

${ }^{8}$ Ces pourcentages ont été obtenus auprès des autorités du Programme National de Lutte contre la Tuberculose au Cameroun. II convient de souligner qu'à côté de cette moyenne nationale de $13 \%$ de malades perdus de vue, certaines régions du pays connaissent des taux davantage préoccupants. La région de l'Est connaît par exemple un taux de PDV de $20 \%$, la province du Sud-Ouest $16 \%$, la ville de Yaoundé 18\%.L'on peut lire aussi utilement Tantchou Yakam, J.C., Comment naissent les politiques verticales de santé en Afrique? Réintégrer le passé et le présent à partir de la lutte contre la trypanosomiase et la tuberculose au Cameroun, EHESS, Thèse de Doctorat nouveau régime en Anthropologie sociale et ethnologie, 2006, p. 327.

${ }^{9}$ CDT signifie Centre de Diagnostic et de Traitement de la TB. Le Cameroun en compte aujourd'hui 189, repartis dans plusieurs hôpitaux du pays.
} 
de lutte sont pourtant visibles ou manifestes. Le pays a créé un Programme National de Lutte contre la Tuberculose $(P N L T)^{10}$ et bénéficie du soutien des partenaires internationaux (Fonds mondial, Union européenne, Coopération allemande...). Les progrès enregistrés sont pourtant «très lents », les succès thérapeutiques sont de plus en plus difficiles et les soignants décrient plutôt une réémergence rapide d'une TB multi résistante. La gratuité des médicaments déclarée et adoptée depuis le $1^{\text {er }}$ Octobre 2004 par le MSP (Ministère de la Santé Publique) semble avoir plutôt compliqué l'accès aux soins. Elle soulève désormais des questions liées à la surpopulation hospitalière ${ }^{11}$ et au suivi réel de la prise en charge efficace des malades tuberculeux. Face à ces discordances entre les progrès institutionnels et les résultats attendus, le sociologue de la santé se pose des questions multiples. Quels sont les logiques, les enjeux et les perspectives manifestes ou latents qui structurent les actions de lutte contre la TB au Cameroun ? Comment se construisent les stratégies, les alliances et les jeux de pouvoir entre les différents groupes d'acteurs impliqués dans les programmes antibuberculose au Cameroun? Quels sont les points de résistance, les zones d'incertitude et les possibilités d'action contrôlés par les différents acteurs ? Quels sont les réseaux relationnels et les processus de soutien mobilisés par les malades?

Ce travail s'est principalement donné pour objectif de comprendre les réseaux relationnels et les processus de soutien mobilisés par les malades tuberculeux au Cameroun.

Dans une posture socio-anthropologique construite à partir du modèle « étiologicothérapeutique $»^{12}$ et de l'analyse des réseaux ${ }^{13}$, l'analyse met en exergue dans le

10 Ce PNLT a été créé en 1996 grâce au soutien des organismes internationaux comme l'Union Européenne et la Coopération Allemande.

${ }^{11}$ La gratuité des médicaments a joué un rôle déterminant dans la croissance du taux de fréquentation des malades tuberculeux dans les CDT. Mais, cette politique nationale de la gratuité n'a pas été suivie par les mesures d'accompagnement comme le recrutement du personnel soignant. Au Cameroun, les principaux ratios sanitaires sont encore préoccupants, 01 médecin pour 10000 habitants, 01 infirmier pour 2250 habitants, 01 lit d'hospitalisation pour 770 malades, avec une moyenne de 01 pharmacie pour 62823 patients.

12 Le modèle « étiologico-thérapeutique », propre à l'anthropologie médicale, permet de comprendre à la fois le discours collectif de la maladie ou l'étiologie subjective du malade. Cette perspective de l'anthropologie médicale rend possible une compréhension significative des rapports de sens et des trajectoires thérapeutiques, des affects et des sentiments éprouvés « du dedans » par les malades et qui sont irréductibles à ce qui peut être d'emblée lu « du dehors». Ce modèle anthropologique a permis de comprendre la TB telle que imaginée, fantasmée, représentée, vécue et prise en charge par les acteurs concernés. A propos de ce modèle «étiologico-thérapeutique », lire utilement Laplantine, F., Anthropologie de la maladie, Paris, Payot, 1986.

13 A propos de l'analyse des réseaux, lire utilement Lazega, E., 1998, Réseaux sociaux et structures relationnelles, Paris, PUF. Lire aussi Mercklé, P., 2004, Sociologie des réseaux sociaux, Paris, La 
contexte camerounais, les représentations, les réseaux relationnels, les processus de soutien, les déterminants des trajectoires et des comportements thérapeutiques, les dynamiques sociales ou familiales construites lorsque survient un cas de tuberculose.

Les résultats présentés ici ont été obtenus à partir des observations directes, des entretiens approfondis et à passage répété, des discussions de groupe, des récits de vie et des discussions informelles menés auprès des malades tuberculeux (internés dans les CDT pour la plupart), des responsables des programmes TB et des professionnels des services des CDT au Cameroun. Le choix de ces techniques de collecte justifie la dimension essentiellement qualitative de ce travail. Ces analyses constituent quelques aspects essentiels de notre travail de thèse de Doctorat /Ph.D.

\section{Des constructions sociales divergentes de la maladie et de la contagion}

Les «formes élémentaires » de la maladie construites par les acteurs sociaux sont plurielles et complexes. Les découvertes pasteuriennes qui valorisent l'interprétation ontologique de la maladie n'ont pas occulté les pratiques populaires et les systèmes de soins traditionnels. Pour diagnostiquer et soigner la maladie, nombre de camerounais prennent en compte à la fois les éléments du « microcosmos», du « mésocosmos » et du « macrocosmos» (Sow 1978). La vie, la maladie comme la mort, sont au quotidien expliquées et déterminées par des considérations sociales, cosmologiques et symboliques diverses. L'on peut donc comprendre pourquoi un cas de tuberculose est un événement qui peut engager les membres de toute une famille.

Les spécialistes de la biomédecine relèvent par exemple parmi les signes cliniques de la tuberculose, les quintes de toux avec une expectoration entachée de sang. Or, nombre de personnes ont une interprétation contraire à cette vision biomédicale. Une toux grasse avec une présence du sang est aussi interprétée chez les Ngyemba $^{14}$ par exemple, comme un indicateur de l'action maléfique des vampires sur les poumons de la victime.

Découverte. Lin, N., 1995, «Les ressources sociales : une théorie du capital social », in Revue française de sociologie, XXXVI, pp. 685-704.

${ }^{14}$ Les Ngyemba constituent un sous-groupe de l'ethnie Bamiléké à l'Ouest-Cameroun. Ils occupent les versants Est des monts Bamboutos et se regroupent autour des chefferies de Bangang, Batcham, Bamougong, Balatchi et Balessing. 
«Lorsque le diagnostic moderne ou un examen de radio révèle des blessures fraîches ou cicatrisées sur le foie, les poumons, les reins, la famille de la victime indexe en priorité l'action du vampire. La tuberculose, le cancer du foie ou des poumons, pour les familles, ne sont pas toujours compris dans leur seule explication rationnelle. Dans l'imaginaire social, le diagnostic biomédical peut être banal, la cause vraie pouvant être extérieure à l'organisme malade. » (Djouda Feudjio 2004: 46)

Certaines familles pensent qu'à travers ses stratégies maléfiques et ses rituels invisibles, le sorcier peut établir un «contact métaphorique » avec la victime, attirer à distance son âme, capter son ombre, « manger » ses organes, soutirer son principe vital et le détruire. Ce qui entraîne un état morbide qui peut se manifester par une la tuberculose. La catégorie causale -sorcellerie- occupe une place importante dans l'explication du malheur au Cameroun. Les acteurs sociaux inscrivent les causes de la maladie aussi bien dans le social et le culturel que dans le biologique. La sorcellerie est une grande constante de la culture camerounaise contemporaine; le mal vient toujours de l'extérieur et demeure l'affaire de la communauté (Ela 1994 ; Hebga 1982).

Les malades tuberculeux et leurs réseaux relationnels n'échappent donc pas à ces «formes élémentaires » de la maladie. Beaucoup de cas de tuberculose sont ainsi socialement interprétés comme étant une conséquence d'une dysharmonie entre l'individu et la sphère $d u$ «microcosme», du «mésocosme» ou du «macrocosme ».

Les ancêtres, les sorciers, l'environnement social sont considérés comme étant de potentielles causes de la maladie. Comme le souligne Laplantine (1986:75) «lorsque la « famille », «la société », « les génies », « les ancêtres », « les sorts » sont appréhendés comme des entités morbifiques, la maladie n'apparaît plus du tout comme étant de l'ordre de l'altération, mais de l'altérité ; de l'invasion, l'effraction (...) ». La tuberculose peut être ainsi un signe, le signe qu'un interdit a été violé ou une règle oubliée. Elle est un appel à l'ordre, un message qui signale que quelque chose d'anormal s'est passée dans la dynamique du groupe familial ou social, qu'une rupture s'est produite (Hountondji 1994: 25). L'état morbide du tuberculeux devient ainsi l'indicateur d'un mal qui frappe le lignage ou la communauté. II est considéré comme le symptôme de «ce qui ne va pas au village », une manifestation directe ou indirecte d'un désordre cosmique, un 
symptôme d'un malaise social, d'une situation conflictuelle à lire ou à déchiffrer (Tsala Tsala 1989 : 110).

Ces constructions profanes de la TB s'observent davantage dans les considérations sociales de ce qu'il convient d'appeler « contagion » ou maladie contagieuse.

\section{La contagion ou la transmission de la TB: Entre les considérations biomédecines et les constructions sociales}

La contagion ou la transmission de la maladie telle que admise dans le contexte biomédical n'a pas toujours la même signification pour les acteurs sociaux profanes. Alors que la biomédecine rapporte la contagion aux substances (salive, morve, urine, sueur, lait maternel, sperme, larme, sang...) transmissibles d'un malade à une personne saine, les profanes n'ont pas toujours cette même construction de la réalité d'une maladie contagieuse ou d'une personne pouvant transmettre la maladie. Une maladie considérée comme biologiquement contagieuse ne l'est pas nécessairement dans toutes les sociétés africaines où les significations sociales de la contagion apparaissent bien diverses ${ }^{15}$. Les observations menées auprès des malades de la TB montrent que les considérations sociologiques de cette maladie sont plurielles. Certains malades dont la TB a été cliniquement déclarée ne sont pas toujours considérés comme souffrant d'une maladie potentiellement dangereuse ou contagieuse. Les proches de cette catégorie de tuberculeux socialement non contaminant cherchent l'origine de la maladie dans les registres naturel, social, sorcier ou magico-religieux. Ils continuent de soutenir leur malade sans prendre en compte les mesures préventives définies dans le champ biomédical. Ils ne prennent donc pas des «attitudes d'évitement » vis-à-vis des crachats, de la salive, des morves ou des éternuements du malade.

Le collectif dirigé par Bonnet et Jaffré (2003) insiste sur le fait qu'en Afrique de l'Ouest aussi, précisément chez les Haoussa et les Songhay-Zerma au Niger, les Dogon et les Senufo au Mali, les Soso en Guinée, la perception et l'acceptation de la contagion d'une maladie donnée sont variables et socialement déterminées. Les liens entre les idées d'une maladie contagieuse et les attitudes de prévention sont complexes, sans « correspondances univoques » (Jaffré $2003: 56$ ).

\footnotetext{
${ }^{15}$ Bonnet, D. et Jaffré, Y. «Transmissions, préventions et hygiène en Afrique de l'Ouest, une question anthropologique », in Bonnet, D. et Jaffré, Y. (dir de), Les maladies de passage, Paris, Karthala, p. 7.
} 
En réalité, un malade potentiellement contagieux d'un point de vue de la biomédecine, ne l'est pas toujours dès lors qu'on prend en compte les considérations sociologiques ou anthropologiques de la contagion et de la maladie considérée. Ici, la contagion peut être construite autour même de la dimension relationnelle entre les individus. Un contact entre deux personnes certes en bonne santé, peut être socialement jugé impur, souillé, contagieux. Un contact social peut être ainsi morbide ou mortifère alors même que les sujets eux-mêmes ne sont pas biologiquement malades. Un homme dont la femme meurt en état de gestation est par exemple socialement considéré comme pouvant transmettre la maladie. Un individu qui a été le premier sur le site d'une mort par pendaison est désormais, s'il n'est pas «lavé », susceptible de transmettre la maladie. Il est donc socialement considéré comme étant contaminé et contaminant.

Dans le processus social de contamination, la contagion n'est donc pas toujours biologique ; même la violation d'un « espace intime » ou d'un « espace interdit » peut transmettre à l'individu une impureté, une maladie contagieuse. Certains espaces sociaux comme des carrefours, les lieux occultes (forêt sacrée, point de chute d'eau...) sont socialement considérés comme dangereux, comme contaminant. La discussion sur la signification du phénomène de contagion ou de transmission apparaît donc complexe. La matérialité morbide n'est pas informe et les expressions pathologiques constituent des indices complexes et souvent trompeurs, notamment lorsqu'ils doivent être interprétés dans un lexique populaire ne disposant pas de l'ouverture d'intelligibilité biomédicale. L'ensemble des représentations populaires de la transmission de la maladie résulte de la rencontre de plusieurs séries de matérialités et de contraintes: celle du corps et des affections morbides prévalentes en une région, celle des mots, des catégories conceptuelles et des savoirs dont disposent une langue et une population à un certain moment de leur histoire ${ }^{16}$.

Ces considérations sociales de la maladie et de la contagion influencent les comportements et les pratiques préventives développées par les acteurs profanes.

\section{Des constructions sociales de la TB au choix des trajectoires thérapeutiques}

\footnotetext{
${ }^{16}$ Jaffré, Y. «Transmissions, prudences et préventions en pays Mande », in Bonnet, D. et Jaffré, Y. (dir de), Les maladies de passage, Paris, Karthala, p. 29.
} 
Les logiques qui structurent la trajectoire thérapeutique des malades de la TB au Cameroun sont d'une complexité réelle. Les observations de terrain montrent que lorsque l'individu manifeste les signes cliniques d'un état morbide (toux, fièvre...), la maladie est généralement «banalisée » au début. Le malade ou ses proches, l'inscrit dans un premier temps, dans le registre des maladies « naturelles » ou «passagères». Ici, la thérapie appropriée est l'automédication ${ }^{17}$. Le malade s'efforce, de sa propre initiative ou en fonction des conseils de son réseau relationnel, de prendre un médicament, sans prescription médicale, sans ordonnance. Cette pratique d'automédication regroupe indifféremment l'automédication par les remèdes traditionnels (plantes, feuilles, herbes, écorces...) et les remèdes conventionnels (comprimés...). Lorsque la maladie est « rebelle » à ces pratiques thérapeutiques locales ou profanes, le malade ou son entourage familial pense à une réorientation du diagnostic et des choix thérapeutiques. Soit le malade et ses proches s'orientent vers un centre de santé ou un hôpital, soit ils pensent à une consultation des nganga ${ }^{18}$ pour rechercher la « cause antécédente » ou le «pourquoi ultime» de la maladie. Le nganga trouvera des réponses aux préoccupations du type : Quelle est la cause réelle ou sous-jacente de la maladie ? Pourquoi telle cause au lieu de telle autre ? Pourquoi la maladie a-t-elle frappé tel individu au lieu de tel autre ? Pourquoi la maladie persiste ou est-elle rebelle ? Dans quelles circonstances (lieu, heure...) la maladie est-elle apparue?

Lorsque les réponses à cette «consultation traditionnelle» ou à ces soupçons trouvent leur signification dans l'irrationnel ou dans la logique cosmique, le malade et son réseau relationnel se retrouvent face à des choix stratégiques qui les engagent dans des «convocations thérapeutiques du sacré » (Massé et Benoist 2002), qui leur font osciller entre le visible et l'invisible, le sens et le non sens, le réel et l'imaginaire (Tsala Tsala 2002).

Dans un contexte où la tuberculose de l'individu peut être la maladie de la communauté (Dan Lantum 1985), retrouver un consensus sur le chemin thérapeutique du malade n'est pas toujours un acquis. La trajectoire thérapeutique suivie peut n'être pas celle voulue par le malade mais celle choisie par son réseau relationnel.

\footnotetext{
17 Il s'agit d'une administration de soi-même ou par soi-même de médicaments, de substances thérapeutiques pour satisfaire à une indication déterminée ou pour produire telle ou telle modification dans la structure ou dans les fonctions de l'organisme. Lire utilement Nelly Molina, L'automédication, Paris, PUF, 1988.

${ }^{18}$ Nous empruntons le mot nganga à De Rosny pour désigner les devins dans la médecine traditionnelle africaine. Lire utilement De Rosny, E. , L'Afrique des guérisons, Paris, Karthala, 1992.
} 
Lorsque les membres du réseau relationnel du malade ne sont donc pas suffisamment informés sur les questions relatives à la tuberculose, ce dernier ne retrouve le chemin de l'hôpital qu'après plusieurs échecs thérapeutiques. Le malade se retrouve ainsi pris en «ballottage», dans une sorte $d^{\text {' } ~ « a v e n t u r e s ~}$ thérapeutiques » ou de «quête thérapeutique » où les soins se font « en rond » (Janzen 1978; Mbadinga 2002), où I'on peut passer du praticien au psychothérapeute, du prêtre exorciste aux « églises de guérison » ou du guérisseur aux rituels ancestraux. On a ainsi des malades tuberculeux «nomades » qui se soignent « au pluriel » et ne reviennent vers I'hôpital que «tardivement » (Noeske et Nkamssé 1998 ; Eliwo Akoto 2002 ; Monteillet 2005), dans la phase chronique ou terminale.

Les membres des réseaux de support prennent généralement les plus importantes décisions dans les choix thérapeutiques. Ceci est dû au fait qu'ils sont aussi dans la plupart des cas, les personnes qui déploient les ressources financières nécessaires pour une prise en charge durable du malade. Sans leur concours, certains malades seraient dans l'impossibilité de se rendre à l'hôpital ou de payer le coût (transport, médicament, nutrition...) de leurs soins de santé.

Nombre de malades en Afrique ne jouissent pas d'une réelle autonomie dans le choix de leur trajectoire thérapeutique. L'une des croyances encore partagées en Afrique porte sur la place insignifiante de l'individu, sur la dépendance du sujet par rapport à la conscience collective. La volonté individuelle est amoindrie par l'autorité de la communauté. L'individu est considéré comme ne pouvant se réaliser totalement ou pleinement sans la solidarité agissante de son groupe. Voilà pourquoi la maladie d'un individu, loin d'être un «fait isolé », concerne aussi ses cousines, ses tantes, ses parents, ses grands-parents, ses amis et ses voisins. Chacun manifeste sa contribution physique, morale, financière ou matérielle pour soulager le membre malade, pour l'aider à choisir une thérapeutique adéquate. « La maladie d'un membre est la maladie de la communauté entière, laquelle n'est pas un rassemblement de personnes uniquement au point de vue numérique, mais une mise en commun d'amour, de vie, de santé et de bonheur » (Dan Lantum $1985: 420)$.

Il y a donc généralement un débat collectif qui est mené entre parents, proches, voisins et amis, pour choisir le chemin sanitaire du malade. Entre le malade, sa famille et son entourage, il y a un «jeu des proximités » (Bonvalet et Maison 1999). Le débat socio-familial prend en compte à la fois les considérations économiques et socioculturelles. Lorsque la maladie est interprétée par exemple 
comme une rupture d'interdit, une manifestation d'un conflit, c'est la famille qui organise les thérapies sous forme de «fêtes», de «réunions familiales » qui constituent l'occasion de dissoudre les tensions individuelles et collectives à travers des confessions publiques, des purifications, des «danses» symboliques, des sacrifices et des réconciliations (Fotso Djemo 1982 :95).

Comme le souligne davantage Schmitz (2006:15), «les choix thérapeutiques s'inscrivent à la fois dans un habitus familial et dans un système de soins local. »; « les choix thérapeutiques ne sont pas isolés mais ont une histoire, celle des corps de la famille et des recours antérieurs » (Raineau (2006:62).

\section{A propos d'une tuberculose socialement contagieuse}

Dans les communautés et les centres de prise en charge visités, les observations montrent que les individus ne développent des « attitudes d'évitement » vis-à-vis de leur malade qu'à partir du moment où ils inscrivent sa maladie dans le champ des pathologies socialement contagieuses.

Un malade tuberculeux qui n'est pas socialement considéré comme contagieux, ne connaît pas autour de lui des «attitudes d'évitement» ou la stigmatisation. Son entourage ou ses proches le gardent, le soutiennent, minimisent et banalisent les risques de contagion connus sur le plan médical. Même s'il souffre d'une tuberculose multi résistante, le risque de contagion, tant qu'il n'est pas socialement admis ou considéré, peut être minimisé au profit des contacts plutôt chaleureux, moins violents ou moins stigmatisants. La stigmatisation d'un malade tuberculeux apparaît donc n'être qu'une simple construction sociale. La peur, le rejet, la méfiance de l' « Autre mortifère » ou d'un «tuberculeux contagieux » devient donc sociologiquement relatif ou contextuel. Un malade TB considéré comme étant contagieux dans un contexte socioculturel « $A$ » ne l'est pas forcement s'il se retrouve dans un autre univers social «B». Cette discordance entre les considérations biomédicales et les constructions sociales de la maladie pose de réelles difficultés quant à la rupture de la chaîne de transmission de la TB. Les sujets contacts qui minimisent ou banalisent le risque de contagion s'exposent pourtant à une contamination cliniquement prouvée. Si les campagnes de sensibilisation ou de prévention de la TB ne sont pas contextualisées, elles peuvent donc avoir moins de chances dans leur applicabilité ou dans leur acceptabilité sociale. Les conduites de prévention ne peuvent pas être des constantes mais des variations correspondant à des adaptations aux contraintes sociales multiples. 


\section{A propos des malades tuberculeux internés dans les Centres de Diagnostic et de Traitement (CDT)}

5.1. La décision d'aller à l'hôpital : un choix relationnel ou motivé

Les observations dans les CDT font constater que nombre de malades internés ou en phase de traitement ambulatoire se sont retrouvé à l'hôpital sous l'influence directe ou indirecte d'un soutien en milieu hospitalier considéré. Certains s'y sont retrouvés parce qu'ils y connaissaient un personnel soignant ou parce qu'ils ont été « confiés » ou « recommandés » par un membre de leur réseau relationnel. Ce réseau a été dans la plupart des cas, constitué d'un soignant ou d'un personnel d'appui membre de la famille du malade, d'un parent, d'un ami ou d'une connaissance, qui devenait un intermédiaire, exerçait l'influence nécessaire pour faciliter la prise en charge du patient. A partir des logiques de la théorie des ressources sociales, l'on peut donc penser avec Lin (1995:691) qu' " aussitôt que l'acteur entre en relation avec un contact ayant de meilleures ressources, ses chances augmentent de voir ces ressources directes ou indirectes du contact l'aider à atteindre le but de son action ».

Dans un contexte de crise économique où la pauvreté a fragilisé de nombreuses familles camerounaises, la négociation de nouveaux réseaux de sociabilité apparaît aujourd'hui être une véritable stratégie mobilisée par les acteurs sociaux pour assurer une relative sécurité sociale et sanitaire. Dans le champ hospitalier, les malades tuberculeux mobilisent ainsi ouvertement les relations familiales, amicales ou professionnelles. Ces nombreuses stratégies constituent, pour les malades, ce que Forse (2000:288) appellerait des «créances» ou des «investissements relationnels ». Dans un centre de prise en charge donc, confessionnel, privé laïc ou public soit-il, plus un malade détient des «créances », plus il détient de privilèges, de facilités et de possibilités dans son itinéraire intra hospitalier.

Même dans les CDT, les malades TB internés pour la phase intensive de traitement ou qui reviennent pour les soins en ambulatoire, comptent sur leur solidarité pour lutter contre certains stigmates dont ils font l'objet.

5.2. Contre les stigmates : une solidarité agissante entre les malades tuberculeux

Aujourd'hui encore, le terme stigmate ou son dérivée stigmatisation, s'emploie en un sens proche du sens littéral originel qu'on lui donnait dans la tradition grecque ${ }^{19}$.

\footnotetext{
${ }^{19}$ Dans la Grèce antique, le stigmate désignait des marques corporelles gravées sur le corps au couteau ou au fer rouge pour donner une identité discriminante à des personnes qui présentaient quelque chose
} 
Cette approche originelle qui associe le stigmate à un discrédit ou à une exclusion est bien applicable dans le contexte hospitalier camerounais où bien de malades tuberculeux, loin d'être considérés comme des malades ordinaires ou « normaux », sont socialement et médicalement classés et catégorisés avec des « attributs» (Goffman 1975) discriminants. La tuberculose de l'individu, contagieuse, apparaît être un «attribut » qui le rend différent des autres malades accomplis ou ordinaires, et fait de lui un malade dangereux, mauvais, vicié, amputé, avec une différence fâcheuse ou honteuse. Le malade tuberculeux diagnostiqué ou « découvert » devient pour certains, un corps souffrant, un corps contaminé et mourrant.

Stigmatisé de part son isolement et son pavillon hospitalier étiqueté comme un lieu contaminé et contaminant, le malade doit faire face aux soupçons, à la dépression, à l'angoisse et même au désarroi. Pour lutter contre les étiquettes offensantes, la plupart des malades tuberculeux recherche d'abord un premier soutien auprès des autres malades de la tuberculose. Le nouveau malade dépisté « se réfugie » auprès des autres tuberculeux qui sont ses « semblables ».

Pour les malades tuberculeux qui se trouvent être abandonnés au CDT ou stigmatisés par leurs proches (parents, collègues, amis...), la nostalgie de l'environnement familial ou professionnel reste prégnante mais, les observations montrent que le nouveau malade est aussi très tôt accueilli dans la famille hospitalière des malades tuberculeux. II se crée une sorte de distance sociale entre lui et son environnement social habituel mais, il gagne de nouvelles relations amicales qui se construisent en milieu hospitalier et lui apportent amour, soutien, sympathie et réconfort.

Ce besoin de sécurité ou de réconfort recherché auprès des autres malades tuberculeux par un nouveau patient stigmatisé se comprend d'un point de vue sociologique, surtout lorsqu'on reconnaît avec Goffman (1975) que les individus ayant le même stigmate ont tendance à se regrouper pour former une catégorie. Celle-ci devient un espace de liberté, un espace d'engagement ou de soutien mutuel.

En réalité,

d'inhabituel ou de détestable. Celui qui portait des stigmates était soit un esclave, un criminel ou un traître, bref, un individu frappé d'infamie, d'impureté et qu'il fallait éviter, surtout dans les lieux publics. Lire utilement, Goffman, E., Stigmate. Les usages sociaux des handicaps, Paris, Minuit, 1975, p. 11. 
«Dans les nombreux cas où la stigmatisation de l'individu s'accompagne de son entrée dans un établissement de garde tel qu'une prison, un sanatorium ou un orphelinat, une grande partie de ce qu'il apprend quant à son stigmate lui est transmis dans l'intimité des contacts prolongés qu'il a alors avec ceux qui sont en train de se transformer en ses compagnons d'infortune » (Goffman 1975 :51).

La solidarité construite entre anciens et nouveaux malades tuberculeux leur permet de se prendre mutuellement en charge. Ils se racontent sans crainte leurs « aventures thérapeutiques » et leurs récits de vie, ils se posent ensemble des questions relatives à leur état morbide, à leur observance thérapeutique, à leur attitude face au traitement ou face au personnel soignant, bref, ils se confient et se libèrent mutuellement. Ces interactions hospitalières se construisent pendant les jeux divers (jeux de cartes, du damier) ou pendant les moments de la « cuisine ${ }^{20}$ ou du repas (très souvent partagé). Entre ces malades, internés pour la même cause, il y a une réelle « acceptation » de leur stigmate commun, pourtant, face à l'étranger qui arrive dans le cadre hospitalier, la stratégie adoptée par ces malades est plutôt celle d' « évitement». Face aux personnes «normales » ou saines, nombre de malades développent des stratégies de dissimulation et de camouflage. Ils évitent de révéler la nature de leur mal, ils ne montrent pas toujours leurs médicaments à tous de peur qu'ils ne les reconnaissent ; leur carte de traitement et leur carnet de soins sont soigneusement dissimulés.

II convient de relever que cette stigmatisation et ses stratégies de dissimulation ne commencent que lorsque les proches du malade inscrivent sa pathologie dans le registre des maladies dangereuses ou contagieuses.

\section{Des malades TB entre logiques hospitalières et logiques profanes}

Les données ethnographiques montrent que nombre de malades camerounais sont orientés, guidés, suivis, conduits et même manipulés sur le chemin des soins par leurs réseaux familiaux ou relationnels. Dans le champ hospitalier, il est apparu que ces personnes intermédiaires ou «gardes malades» qui influencent les pratiques du malade, ne sont pourtant pas toujours considérées. Leurs actions et leur utilité restent invisibles dans le choix des professionnels hospitaliers. Ces acteurs du réseau de support sont dans certains cas exclus des lieux de soins. Pour visiter leur malade, ils doivent très souvent se conformer aux règles exigeantes (horaires de

\footnotetext{
${ }^{20}$ Dans la plupart des CDT au Cameroun, les responsables laissent la possibilité aux malades tuberculeux d'aménager dans des coins isolés de l'hôpital, une cuisine qui leur permet d'assurer au quotidien leur repas. Ceci est dû au fait que l'éloignent géographique de certains malades de leur village ne leur permet pas de bénéficier chaque jour des repas préparés dans leur famille.
} 
visite, nombre de visiteurs limité...) de l'organisation hospitalière considérée. Pourtant, beaucoup veulent avoir un droit de regard sur ce qui est dit ou fait sur leur malade par le personnel soignant. Cette distanciation entre les logiques hospitalières et les logiques sociofamiliales met le malade dans une situation ambiguë où il est en «ballottage » entre un suivi médical spécialisé et une pression familiale parfois contraignante. Les cas où certains « gardes malades » choisissent de quitter le CDT avec leur malade, pour d'autres recours thérapeutiques peuvent ainsi être compris comme l'un des effets pervers de ces logiques de soins contraires. L'orientation du malade vers d'autres recours de soins peut donc être comprise comme une manière pour les «gardes du malade » de briser ou de rompre la distanciation et le manque de dialogue avec les soignants. Certains malades tuberculeux se retrouvent ainsi dans une sorte de mobilité spatiale aux logiques et aux conséquences multiples.

\section{La mobilité spatiale des malades tuberculeux : logiques et conséquences}

Dans les représentations sociales en général, la TB reste considérée comme une «maladie honteuse », une «maladie des pauvres». Les données de terrain font observer que pour certains malades qui s'estiment être des «élites ${ }^{21}$, souffrir d'une maladie comme la TB apparaît comme un discrédit social. Ces malades d'une certaine identité sociale se retrouvent dans des trajectoires thérapeutiques complexes, à vrai dire indescriptibles. Le Dr. Jurgen, rapportant certains cas à lui révélés par un médecin spécialiste camerounais, le professeur Kuaban, souligne que «les gens cherchent à faire rendez-vous avec lui plutôt la nuit ou chez lui à la maison. Certaines personnalités refusent le diagnostic de la tuberculose en disant que moi avec mon rang social je peux attraper la tuberculose !».

La TB pose donc ainsi des questions liées aux jeux de statut. Les malades qui ont un meilleur capital économique, social ou culturel créent autour d'eux d'autres réseaux de soins qui ne sont pas enregistrés dans les circuits formels mais qui sont entretenus par les soignants. L'on a vu au Cameroun, des malades « nantis » qui arrivent dans les CDT, restent dans le parking, téléphone à un soignant qui leur

\footnotetext{
21 Le concept $d^{\prime}$ " élites » renvoie ici à plusieurs catégories d'acteurs. L'on y intègre les élites traditionnelles qui jouissent d'une influence sociale construites dans les croyances, les élites technocratiques dont le pouvoir est bureaucratique, les élites de propriété qui disposent des multiples biens/capitaux sociaux ou des élites symboliques qui incarnent certaines valeurs et sont les prototypes de certaines manière de vivre ou de faire. Pour comprendre davantage cette catégorisation des élites, I'on peut lire utilement Rocher, G, Introduction à la sociologie générale, livre 3, le changement social, Paris, Editions $\mathrm{HMH}, 1968$, p. 136-137.
} 
prépare l'ordonnance ainsi que les médicaments, ceci pour des retombées inavouées (cadeaux, argent...). C'est le soignant qui parcourt ainsi l'itinéraire intrahospitalier à la place de «son» malade, en gardant l'anonymat et la confidentialité nécessaires.

Les données de terrain montrent que bien de malades TB, même dans un contexte de la décentralisation et de la gratuité des médicaments contre la TB, ont une préférence plutôt pour les centres de traitement localisés dans des régions éloignées de leur lieu de résidence. Ils abandonnent les services de prise en charge prévus pour desservir leur localité pour s'insérer dans des réseaux de soins de longue distance, dans des villes ou localités où leur identité est méconnue et où ils pourront bénéficier de la confidentialité ou de l'anonymat.

Ces multiples pratiques de dissimulation, structurées à l'origine par la stigmatisation sociale, posent de réelles difficultés dans le processus de prise en charge des malades. II est pratiquement difficile de suivre convenablement l'observance thérapeutique chez un malade de longue distance. Les malades enregistrés dans les circuits informels ou privés des soignants créent des réseaux de circulation clandestine de médicaments. Ce camouflage des malades compromet les chances d'application ou d'acceptation d'une politique DOTS pour tous les malades TB.

\section{Discussion et conclusion}

Ce travail interrogeait principalement les réseaux relationnels et les processus de soutien mobilisés par les malades tuberculeux au Cameroun. II convient de relever ici que les déterminants et les logiques qui expliquent les choix thérapeutiques des malades camerounais tuberculeux sont complexes. La trajectoire thérapeutique du malade ne dépend pas toujours de sa claire volonté mais aussi et même surtout de son réseau relationnel (cousines, tantes, parents, amis, voisins...). Celui-ci joue un rôle prépondérant dans le choix et l'organisation de la thérapie. Les membres du support décident non seulement du moment où le malade doit visiter I'hôpital ou le guérisseur, mais, ils jouent un rôle crucial pour ce qui est de la détermination du diagnostic, de l'acception sociale du malade, de ses soins et de sa réintégration dans le milieu socio-familial. Même la dimension contagieuse de la maladie, établie dans la biomédecine, s'inscrit dans une complexité de croyances étiologiques, marquée par une contextualisation du sens de la contagion. Une tuberculose considérée comme biologiquement contagieuse ou dangereuse ne l'est pas nécessairement dans l'environnement social du malade, où elle peut être rapportée 
à un fait magico-religieux, et provoquer des attitudes et des pratiques thérapeutiques contraires. Les choix thérapeutiques ne sont donc pas isolés, ils ont une histoire et sont déterminés par un habitus familial ou par les rapports de force socio-familiaux.

\section{Bibliographie}

Aubey, P. (2004). «La tuberculose à I'heure du Sida, actualités 2004 », Médecine tropicale, disponible sur http://medecinetropicale.free.fr/.

Berçon, R. et Kuaban, C. (1988). Résistances de mycrobactérium tuberculosis complex aux principaux antibacillaires à Yaoundé.

Bonnet, D. et Jaffre, Y. (2003). «Transmissions, préventions et hygiène en Afrique de l'Ouest, une question anthropologique ». Bonnet, D. et Jaffre, Y. (dir de), Les maladies de passage, Paris, Karthala, pp.5-28.

Brown, P. (1993). «Une maladie bien vivante ». Santé du monde, no 4, juilletaoût, p.4-5.

Caprara, A. (2000). Transmettre la maladie. Représentations de la contagion chez les Alladian de la Côte d'I voire, Paris, Karthala.

Carricaburu, D. et Menoret, M. (2005). Sociologie de la santé, Paris, Armand Colin.

De Rosny, E. (1974). Ceux qui soignent dans la nuit, Yaoundé, Clé.

De Rosny (1992). L'Afrique des guérisons, Paris, Karthala.

Djouda Feudjio, Y. B. (2004). La symbolique de la maladie et la pratique du «blindage » traditionnel dans le groupement Bangang (Ouest-Cameroun, Université de Yaoundé I, Département de Sociologie-Antrhopologie, mémoire de Maîtrise en Sociologie.

Dujardin, B. (2003). Politiques de santé et attentes des patients. Vers un dialogue constructif, Paris, Karthala.

Eliwo Akoto, M. (2002). Se soigner aujourd'hui en Afrique de l'Ouest: Pluralisme thérapeutique entre traditions et modernité (Bénin, Côte D'ivoire et Mali), Les cahiers de L' IFORD $n^{\circ} 27$.

Favret-Saada. (1977). Les mots, la mort, les sorts, Paris, Gallimard.

Forse, M. (2000). « Les relations sociales comme ressources ». Cabin, P. et Dortier, J-F., (eds), La sociologie. Histoire et idées, Paris, Sciences Humaines, pp. 287-293.

Fotso Djemo, J. B. (1982). Le regard de l'autre. Médecine traditionnelle africaine, Paris, Silex.

Goffman, E., (1975). Stigmate. Les usages sociaux des handicaps, Paris, Minuit.

Gruénais, M-E. (1990). «Le malade et sa famille. Une étude de cas à Brazzaville». Fassin, D et Jaffré, Y. (dir de), Societés, développement et santé, Paris, Ellipses/AUPELF, pp. 227-242. 
Hebga Meinrad, P. (1982). Sorcellerie et prière de délivrance, Paris-Abidjan, Inadès-Présence africaine.

Hountondji, J. P. (sous la direction de) (1994). Les savoirs endogènes, pistes pour une recherche, Paris, Karthala.

Jaffre, Y. (2003). «Transmissions, prudences et préventions en pays Mande ». Bonnet, d. et Jaffre, Y. (dir de), Les maladies de passage, Paris, Karthala.

Jaffre, Y. et Olivier De Sardan, J.-P. (dir. de). (2003). Une médecine inhospitalière, Paris, Apad-Karthala.

Lantum, D. N. (1985). «L'identité culturelle du Cameroun exprimée par la science et la technologie ». L'identité culturelle camerounaise, Actes du colloque de la deuxième semaine culturelle nationale, Yaoundé, Ministère de l'information et de la culture, pp. 416-423.

Laplantine, F. (1986). Anthropologie de la maladie, Paris, Payot.

Lazega, E. (1998). Réseaux sociaux et structures relationnelles, Paris, PUF.

Lévi-Strauss, C. (1962). La pensée sauvage, Paris, Plon.

Lin, N. (1995). «Les ressources sociales: une théorie du capital social ». Revue française de sociologie, XXXVI, pp. 685-704.

Masse, R. et Benoist, J. (dir de). (2002). Convocations thérapeutiques du sacré, Paris, Karthala.

Mbadinga, S. et al. (2002). «Se soigner en rond: Sur le fond du partage de l'espace thérapeutique en Afrique noire ». Tsala Tsala, J. P. Santé mentale, psychothérapies et sociétés, Vienna, the world council for psychotherapy, pp.89-98.

Monteillet, N. (2005). Le pluralisme thérapeutique au Cameroun. Crises hospitalières et nouvelles pratiques populaires, Paris, Karthala.

Murray, C. J. L. et al. (1990). «La tuberculose dans les pays en développement: importance, stratégies de lutte et coût ». Bulletin de l'Union internationale contre la tuberculose et les maladies respiratoires, vol. 65, no 1, 1990.

Nelly Molina. (1988). L'automédication, Paris, PUF, Collection les champs de la santé.

Retel-Laurentin, A. (1974). Sorcellerie et ordalies ; L'épreuve du poison en Afrique noire ; Essai sur le concept de négritude, Paris, Anthropos.

Sow, I. (1978). Les structures anthropologiques de la folie en Afrique noire, Paris, Payot.

Tantchou Yakam, J.C. (2006). Comment naissent les politiques verticales de santé en Afrique? Réintégrer le passé et le présent à partir de la lutte contre la trypanosomiase et la tuberculose au Cameroun, EHESS, Thèse de Doctorat nouveau régime en Anthropologie sociale et ethnologie.

Tsala Tsala, J. P. (dir de). (2002). Santé mentale, psychothérapies et sociétés, Vienna, the world council for psychotherapy. 
Tsala Tsala, J. P. (1989). De la demande thérapeutique au Cameroun, Rev. De méd. Psychosomatique. 